\title{
Si photonic device uniformity improvement using wafer-scale location specific processing
}

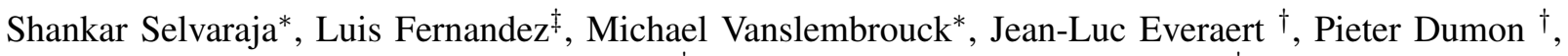 \\ Joris Van Campenhout ${ }^{\dagger}$, Wim Bogaerts*and Philippe Absil ${ }^{\dagger}$ \\ *Photonics Research Group, Ghent University - imec, Ghent, Belgium. \\ ${ }^{\dagger}$ imec, Leuven, Belgium. ${ }^{\ddagger}$ TEL Epion, Billerica, Massachusetts, United States of America.
}

\begin{abstract}
We report two-fold improvement in $\mathrm{Si}$ photonic device uniformity over a $200 \mathrm{~mm}$ SOI wafer through location specific processing. A within wafer thickness non-uniformity of 0.8nm yielding a grating fiber-coupler peak-wavelength nonuniformity of $1.8 \mathrm{~nm}$ is achieved.
\end{abstract}

\section{INTRODUCTION}

Over the years silicon photonics has matured from being a research interest to a commercial reality. Being a high-index contrast material, Si PIC allows compact circuits reducing foot by many orders of magnitude compared to traditional silicaon-Silicon based circuits. The drawback of high confinement when using $220 \mathrm{~nm}$ Silicon on top of $2000 \mathrm{~nm}$ BOX is the high dependence of device response to dimensional variations (thickness, line width and etch depth). For example, a simple device such as a grating fiber coupler will see its peak coupling wavelength shift by $2 \mathrm{~nm}$ for only $1 \mathrm{~nm}$ of silicon thickness variation. This illustrates the important of dimensional control for commercial viability of highly confined Si PICs. Hailing from semiconductor industry, these wafers have within wafer thickness non-uniformity(NU) of $\pm 10 \% \mathrm{~nm}$, which is too large to make matched devices. Hence identifying schemes to address the NU issue for wafer-scale manufacturing process has become more important.

In this paper, we present within wafer grating fiber-chip coupler uniformity improvement through location specific thickness correction using Gas Cluster Ion Beam (GCIB) etching. We also present the effect of the location specific process on the chemical composition and optical quality of $\mathrm{Si}$ surface.

\section{EFFECT OF THICKNESS VARIATION}

Grating fiber-chip couplers are simple yet an essential device in a PIC to couple light in and out of a chip. A grating coupler at $1550 \mathrm{~nm}$ is defined by a linear grating with a pitch of $630 \mathrm{~nm}$ and $50 \%$ fill factor. The grooves were $70 \mathrm{~nm}$ shallow etch in $220 \mathrm{~nm}$ thick Si to create the refractive index modulation [1]. Any deviation in these dimensions would shift the peak wavelength of the coupler. Fig. 1 depicts simulated effect of linewidth, etch depth and thickness variation on the peak wavelength of the coupler. As mentioned earlier, the effect of thickness variation is one order of magnitude higher than the width variation.

\section{EXPERIMENT}

\section{A. Thickness correction process}

Location specific thickness correction is a process though which a predetermined amount of thickness is removed by scanning an ion beam to reach a targeted $\mathrm{Si}$ thickness. The etching ion beam is generated by a $\mathrm{CHF} 3 / \mathrm{O} 2$ plasma and directed toward the wafer [2]. Since our devices are designed for $220 \mathrm{~nm}$ thick silicon we need to increase first the layer thickness by using epitaxy to $245 \mathrm{~nm}$ in order to eventually reach $228 \mathrm{~nm}$ after thickness correction process. After thickness correction, the contamination created by the plasma on the surface is cleaned by a curing process involving thermal oxidation (at $900 \mathrm{C}$ ) and subsequent removal of that layer using buffered hydrofluric acid resulting in final SI thickness of $220 \mathrm{~nm}$. Grating couplers and waveguides were patterned by etching $70 \mathrm{~nm}$ and $220 \mathrm{~nm}$ into Si respectively both using $193 \mathrm{~nm}$ optical lithography and dry etch process. We also fabricated same devices in un-corrected wafer as reference. The chemical and physical property of the Si surface after correction and defect curing process was also done using Xray photoelectron spectroscopy and atomic force microscopy respectively. The optical quality of Si was evaluated through propagation loss measurement in the photonic wire waveguides.

\section{RESULTS AND DISCUSSION}

\section{A. Si thickness uniformity}

Fig. 2 shows Si layer thickness over a $220 \mathrm{~mm}$ wafer before and after the correction process. Using a thickness correction

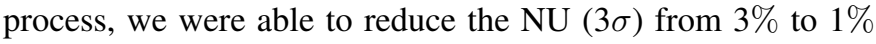
while the range is reduced from $11.5 \mathrm{~nm}$ to $5.1 \mathrm{~nm}$. It has to be noted that defect curing process did not change NU of the Si layer.

\section{B. Post-correction surface and optical quality}

Fig. 3a shows the chemical contamination (C anf F) originating from the plasma source on the Si surface. We clearly observed reduction in contaminants after defect cure process. With AFM, we measured a surface roughness of $0.4 \mathrm{~nm}$, slightly higher than the virgin wafer roughness of $0.1 \mathrm{~nm}$. However, low enough to achieve low-loss waveguides

We measured a loss of $1.77 \pm 0.3$ and $1.46 \pm 0.16 \mathrm{~dB} / \mathrm{cm}$ (Fig. $3 b)$ for corrected and un-corrected wafer. Compared to uncorrected wafer an excess loss of $0.29 \mathrm{~dB}$, which is well within 

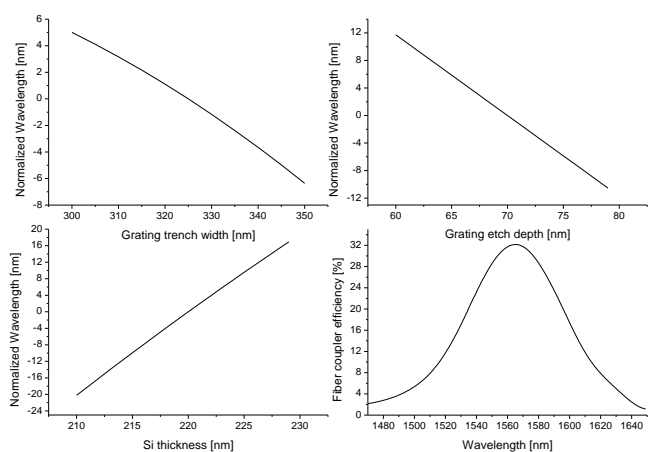

Fig. 1: Effect of dimensional variation on fiber coupler characteristics.

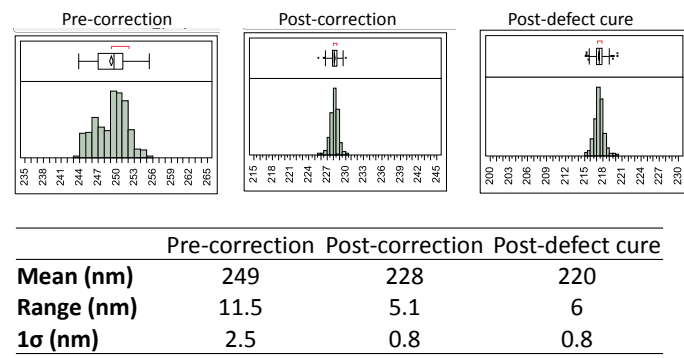

Fig. 2: Si thickness distribution over a 200mm wafer (361 sites).

measurement error margin. Through this low-loss waveguide we confirm that physical and chemical quality of $\mathrm{Si}$ is not degraded due to the correction process.

\section{Grating fiber coupler uniformity}

Uniformity of the grating fiber coupler was extracted from the transmission spectrum of a $2 \mathrm{~mm}$ long waveguide with identical coupler at both the ends of the waveguide. Fig. 4 shows the peak wavelength map of the fiber couplers from thickness corrected and un-corrected wafer. Since the final $\mathrm{Si}$ thickness of the corrected wafer was $217 \mathrm{~nm}$ there is a red shift in the average peak wavelength compared to the un-corrected wafer whose mean Si thickness was $221 \mathrm{~nm}$. Nevertheless, it can be clearly seen that the range and standard deviation of the corrected wafer is 2 fold better than the uncorrected wafer.

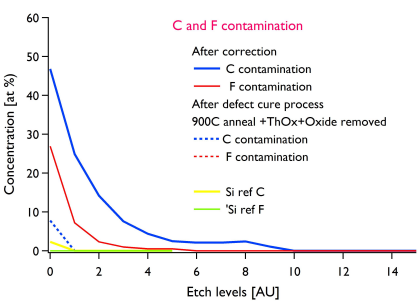

(a)

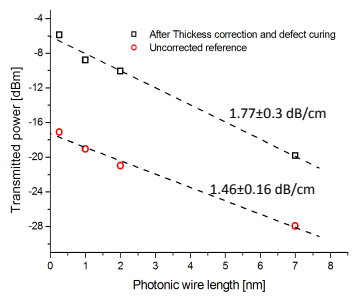

(b)
Fig. 3: (a) Carbon and Fluorine contamination. and (b) Photonic wire loss of corrected and un-corrected wafer.

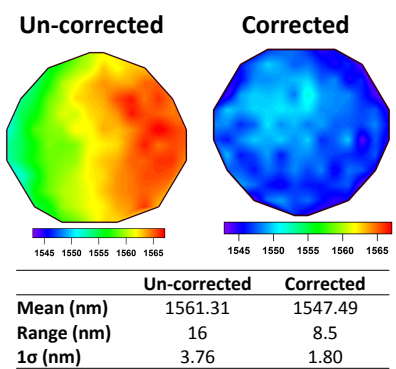

Fig. 4: Grating fiber coupler peak wavelength over a $200 \mathrm{~mm}$ wafer.

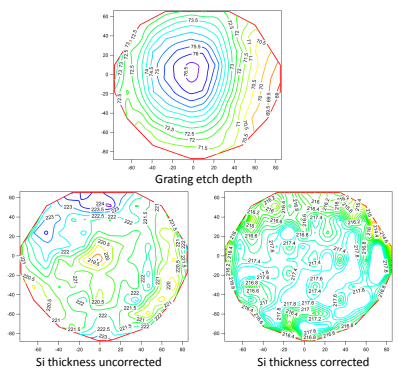

Fig. 5: Etch depth and Si thickness Non-uniformity

We observe an interesting distribution between corrected and un-corrected wafers. In the un-corrected wafer, the peak wavelength has a red shift from east-to-west, while we observe a familiar radial pattern in corrected wafers. Correlating the spectral response with the etch depth and thickness data collected during fabrication (Fig. 5), we observe that overlap of radial yet tilted (east side of the wafer has higher etch depth compared to west) NU of etch depth of the gratings and radial Si thickness NU is causing an east-to-west shift in peak wavelength in uncorrected wafer. The corrected wafers do not have the radial pattern of the silicon thickness which makes etch depth NU emerges clearly from the spectral response. Hence, in corrected wafer etch depth NU is identified as the dominant NU source, while in uncorrected wafer combination of both thickness and etch depth affect device NU. We also show the spectral response correlated well with the simulated spectrum from measured device dimensions.

\section{CONCLUSION}

We have shown a pre-pattern thickness correction using location specific processing though which we achieved $50 \%$ reduction in fiber coupler non-uniformity over a $200 \mathrm{~mm}$ SOI wafer.

\section{ACKNOWLEDGMENT}

The authors wish to thank imec p-line for the fabrication of the silicon photonics wafers. This work was supported by imecs Core Partner Program.

\section{REFERENCES}

[1] D. Taillaert et al., Jpn. J. Appl. Phys., Part 1, vol. 45, pp. 6071-6077, 2006.

[2] I. Yamada et al., Methods Phys. Res., Sect. B, vol. 206, pp. 820-829, 2003 